\title{
A new Glivenko Theorem
}

\author{
Majid Alizadeh ${ }^{1}$, Mohammad Ardeshir², and Wim Ruitenburg ${ }^{3 *}$ \\ 1 School of Mathematics, Statistics and Computer Science, College of Science, University of Tehran, \\ P.O. Box 14155-6455, Tehran, Iran malizadeh@khayam.ut.ac.ir \\ 2 Department of Mathematics, Sharif University of Technology, P.O. Box 11365-9415, Tehran, Iran \\ mardeshir@sharif .edu \\ 3 Department of Mathematics, Statistics and Computer Science, Marquette University, P.O. Box \\ 1881, Milwaukee, WI 53201, USA wimr@mscs.mu.edu
}

\section{Introduction}

Basic Propositional Calculus BPC, which was introduced by Albert Visser in [5], captures a sublogic of Intuitionistic Propositional Calculus IPC which corresponds with modal logic K4 in essentially the same way that IPC corresponds with modal logic S4. In [3] and 4 we introduce Visser algebras (where we named them basic algebras), which correspond with BPC in the same way that Heyting algebras correspond with IPC and that Boolean algebras correspond with Classical Propositional Calculus CPC.

The double negation construction of Boolean algebras from Heyting algebras is well-known. It is natural to consider how closely one can repeat this construction over Visser algebras. Surprisingly the end result still works, although in details we use several new ideas.

Glivenko's Theorem also goes through, but with an interesting reformulation. Given propositional formula $\psi$, define $\xi(\psi):=((\top \rightarrow \psi) \rightarrow \psi) \rightarrow(\top \rightarrow \psi)$. Formulas $\xi(\psi)$ are of interest in their own right, see [4, page 323]. Over IPC, formulas $\psi$ and $\xi(\psi)$ are equivalent. So, in particular, IPC proves $\neg \xi(\perp)$. With Theorem 4.7 we show that for all (sequent) theories $\Gamma \supseteq \mathrm{BPC}$ we have

$\Gamma$ proves $\varphi \rightarrow \xi(\perp) \quad$ if and only if $\quad \Gamma+\mathrm{CPC}$ proves $\varphi \rightarrow \xi(\perp)$.

So if $\Gamma \supseteq$ IPC, then $\Gamma$ proves $\neg \varphi$ if and only if $\Gamma+$ CPC proves $\neg \varphi$ (Glivenko's Theorem).

\section{Boolean Algebras}

For the purposes of this paper we introduce notations $\square a$ for $1 \rightarrow a$, and $x^{a}$ for $x \rightarrow a$. So $\square \square a=1 \rightarrow(1 \rightarrow a)$, and $x^{a a a}=((x \rightarrow a) \rightarrow a) \rightarrow a$. For all terms $t(x)$ built from the defining functions of $\mathfrak{A}=(A, \wedge, \vee, \rightarrow, 0,1)$ and the elements $A$, and for all $x \in A$, we have $x \wedge t(x)=x \wedge t(1)$ (simple substitution). Positive and negative occurrences in formulas and terms are defined in the usual way. If $x$ is only positive in $t(x)$, then $x \leq y$ implies $t(x) \leq t(y)$. If $x$ is only negative in $t(x)$, then $x \leq y$ implies $t(y) \leq t(x)$.

An element $a$ is called Heyting if $\square a=a$. A Visser algebra is a Heyting algebra exactly when all its elements are Heyting.

Proposition 2.1. Let $a$ be an element of Visser algebra $\mathfrak{A}$. Then we have equations $(x \wedge y)^{a} \leq$ $x \rightarrow y^{a} \leq(x \wedge y)^{\square a}$, and $(x \wedge y)^{a a}=x^{a a} \wedge y^{a a}$.

* Corresponding author

N. Galatos, A. Kurz, C. Tsinakis (eds.), TACL 2013 (EPiC Series, vol. 25), pp. 191 194 
Let $a \in A$. An element $x$ is called $a$-regular if $x^{a a}=x$. Let $R^{a}(\mathfrak{A})$ be the set of $a$-regular elements of $\mathfrak{A}$. Clearly we have $\left\{x^{a} \quad: x \in A\right\} \supseteq\left\{x^{a a}: x \in A\right\} \supseteq\left\{x^{a a a}: x \in A\right\} \supseteq$ $\ldots \supseteq R^{a}(\mathfrak{A})$. Since $x$ is positive in $x^{a a}$ and $0^{a a}=\square a$ and $1^{a a}=\square a \rightarrow a$, we also have $R^{a}(\mathfrak{A}) \subseteq[\square a, \square a \rightarrow a]$. The set $R^{a}(\mathfrak{A})$ inherits a partial order from $\mathfrak{A}$.

Proposition 2.2. Let a be an element of Visser algebra $\mathfrak{A}$. Then

1. $x \in R^{a}(\mathfrak{A})$ implies $x^{a} \in R^{a}(\mathfrak{A})$

2. $\square a \in R^{a}(\mathfrak{A}) \quad$ (this is 4, Proposition 2.12])

3. $\square a \rightarrow a \in R^{a}(\mathfrak{A})$

4. $x, y \in R^{a}(\mathfrak{A})$ implies $x \wedge y \in R^{a}(\mathfrak{A})$

Given $a \in A$, define $x \vee_{a} y=(x \vee y)^{a a}$.

Proposition 2.3. Let $a$ be an element of Visser algebra $\mathfrak{A}$. Then

1. $x, y \in R^{a}(\mathfrak{A})$ implies $x \vee_{a} y \in R^{a}(\mathfrak{A})$

2. $x, y \in R^{a}(\mathfrak{A})$ implies $x \vee y \leq x \vee_{a} y$

3. $z \in R^{a}(\mathfrak{A})$ plus $x \vee y \leq z$ imply $x \vee_{a} y \leq z$

4. $x \in R^{a}(\mathfrak{A})$ implies $x \wedge\left(y \vee_{a} z\right)=(x \wedge y) \vee_{a}(x \wedge z)$

Given $a \in A$, define $x \rightarrow_{a} y=x^{a} \vee_{a} y$. Let $\mathfrak{R}^{a}(\mathfrak{A})$ be structure $\left(R^{a}(\mathfrak{A}), \wedge, \vee_{a}, \rightarrow_{a}, \square a, \square a \rightarrow\right.$ a). By Propositions 2.2 and 2.311, this structure is well-defined.

Theorem 2.4. Let a be an element of Visser algebra $\mathfrak{A}$. Then $\mathfrak{R}^{a}(\mathfrak{A})$ is a Boolean algebra.

\section{Boolean Elements and Morphisms}

We have a further characterization of the elements of $R^{a}(\mathfrak{A})$ which allows us to find an idempotent Visser algebra morphism from the 'subalgebra' of $\mathfrak{A}$ on interval $[a, 1]$, onto $\mathfrak{R}^{a}(\mathfrak{A})$.

Proposition 3.1. Let $a$ be an element of Visser algebra $\mathfrak{A}$. Then we have equations $x \wedge x^{a a}=$ $x \wedge(\square a \rightarrow a)$, and $x^{a a a}=x^{a} \wedge(\square a \rightarrow a)$, and $x^{a a a a}=x^{a a}$. So $R^{a}(\mathfrak{A})=\left\{x^{a a}: x \in A\right\}$.

Let $a, b \in A$ be with $a \leq b$. We construct a Visser algebra $\mathfrak{I}^{[a, b]}(\mathfrak{A})$ on interval $[a, b]$ as follows. Define $x \rightarrow_{I} y=(x \rightarrow y) \wedge b$. Define $\mathfrak{I}^{[a, b]}(\mathfrak{A})=\left([a, b], \wedge, \vee, \rightarrow_{I}, a, b\right)$. Clearly $\mathfrak{I}^{[a, b]}(\mathfrak{A})$ is well-defined. The map $\pi_{[a, b]}: x \mapsto(x \wedge b) \vee a=(x \vee a) \wedge b$ is a well-defined map from $A$ onto $[a, b]$. If $b=1$, then $x \rightarrow_{I} y=x \rightarrow y$, so $\mathfrak{I}^{[a, 1]}(\mathfrak{A})$ is clearly a Visser algebra, and is a subalgebra of $\mathfrak{A}$ except for the bottom element.

Proposition 3.2. Let $a \leq b$ be elements of Visser algebra $\mathfrak{A}$. Then $\mathfrak{I}^{[a, b]}(\mathfrak{A})$ is a Visser algebra, and $\pi_{[a, b]}$ is an idempotent bounded distributive lattice morphism from $\mathfrak{A}$ onto $\mathfrak{I}^{[a, b]}(\mathfrak{A})$.

Finally the morphism of primary interest. Let $a \in A$. Define map $\gamma_{a}: A \rightarrow R^{a}(\mathfrak{A})$ by $\gamma_{a}(x)=x^{a a}$. By Proposition 3.1 map $\gamma_{a}$ is well-defined. We are primarily interested in $\gamma_{a}$ with restriction to subdomain $[a, 1]$.

Proposition 3.3. Let $a$ and $b$ be elements of Visser algebra $\mathfrak{A}$. Then we have equations $\left(x^{a a} \vee y^{a a}\right)^{a a}=(x \vee y)^{a a}$, and $(x \rightarrow(b \vee y))^{a} \leq((x \rightarrow b) \vee y)^{a}$, and $(x \rightarrow(a \vee y))^{a}=((x \rightarrow$ a) $\vee y)^{a}$.

Theorem 3.4. Let $a$ be an element of Visser algebra $\mathfrak{A}$. Then $\gamma_{a}$ is an idempotent Visser algebra morphism from $\mathfrak{I}^{[a, 1]}(\mathfrak{A})$ onto $\mathfrak{R}^{a}(\mathfrak{A})$. 


\section{Glivenko Theorems}

Let $\mathfrak{A}$ be a bounded distributive lattice with binary function $x \rightarrow y$ satisfying $x \rightarrow y=1$ for all $x, y \in A$. Then $\mathfrak{A}$ is clearly a Visser algebra. All Visser algebras satisfying $\square 0=1$ can so be obtained from bounded distributive lattices. They belong to the collection of Visser algebras that satisfy the principle of excluded middle $x \vee x^{0}=1$. So the principle of excluded middle is not sufficient to yield just Boolean algebras. Therefore the following is not completely self-evident.

Proposition 4.1. Let Visser algebra $\mathfrak{A}$ satisfy the schema of double negation elimination $x^{00} \leq$ $x$. Then $\mathfrak{A}$ is a Boolean algebra.

For Visser algebra elements $a$, define $\xi(a)=(\square a \rightarrow a) \rightarrow \square a$.

Proposition 4.2. Let a be an element of Visser algebra $\mathfrak{A}$. Then

1. $\xi(a) \wedge(\square a \rightarrow a)=\square a$

2. $\square \xi(a)=\xi(a) \quad$ (this is [4, Proposition 2.11])

3. $x \rightarrow \xi(a)=1$ if and only if $x \leq \xi(a)$

4. $\xi(a) \rightarrow a=\square a \rightarrow a$

Proposition 4.3. Let a be an element of Visser algebra $\mathfrak{A}$. Then

$\square a \rightarrow a \leq x^{a}$ if and only if $x^{a a}=\square a$ if and only if $x \leq \xi(a)$

So the inverse image of $\square a$ under $\gamma_{a}$ is the principal ideal $[0, \xi(a)]$.

Theorem 4.4. Let $a$ be an element of Visser algebra $\mathfrak{A}$, and $\gamma_{a}(x)=x^{a a}$ be the idempotent bounded distributive lattice morphism from $\mathfrak{A}$ onto $\mathfrak{R}^{a}(\mathfrak{A})$. Then $\gamma_{a}^{-1}(\square a)=\{x \in A$ : $\left.x^{\xi(a)}=1\right\}$ and $\gamma_{a}^{-1}(\square a \rightarrow a)=\left\{x \in A: x^{a \xi(a)}=1\right\}$.

Fix a propositional language $\mathcal{L}$. With its presentation in [3] (see also [4, Proposition 2.4]), the Lindenbaum algebra of basic propositional logic BPC is isomorphic in the natural way with the free Visser algebra on the set of propositional letters of $\mathcal{L}$. Sequent theories $\Gamma \supseteq \mathrm{BPC}$ correspond with adding equations between (equivalence classes of) formulas of $\mathcal{L}$. Examples are intuitionistic propositional $\operatorname{logic} \Gamma=\mathrm{IPC}$, which is axiomatizable by schema $T \rightarrow \varphi \Rightarrow \varphi$, and classical propositional logic $\Gamma=\mathrm{CPC}$, which is axiomatizable by schema $(\varphi \rightarrow \perp) \rightarrow \perp \Rightarrow \varphi$, also written as $\neg \neg \varphi \Rightarrow \varphi$. Write $\mathfrak{A}_{\Gamma}$ for the Lindenbaum Visser algebra of $\Gamma$, with elements $[\varphi]_{\Gamma}=\{\psi \in \mathcal{L}: \Gamma \vdash \psi \Leftrightarrow \varphi\}$. Given sequent theories $\Gamma \subseteq \Delta$, the map $\pi_{\Delta}^{\Gamma}:[\varphi]_{\Gamma} \mapsto[\varphi]_{\Delta}$ is a Visser algebra morphism from $\mathfrak{A}_{\Gamma}$ onto $\mathfrak{A}_{\Delta}$. A Visser algebra morphism $\mu: \mathfrak{A} \rightarrow \mathfrak{B}$ induces a congruence on $\mathfrak{A}$ in the usual way by $x \sim y$ exactly when $\mu(x)=\mu(y)$. If $\mathfrak{A}=\mathfrak{A}_{\Gamma}$ for some sequent theory $\Gamma$, then $\Delta(\mu)=\left\{\varphi \Rightarrow \psi:[\varphi]_{\Gamma} \sim[\varphi \wedge \psi]_{\Gamma}\right\}$ is the unique sequent theory containing $\Gamma$ such that $\mathfrak{A}_{\Gamma} /(\sim) \cong \mathfrak{A}_{\Delta(\mu)}$ by the usual isomorphism $\left[[\varphi]_{\Gamma}\right]_{\sim} \mapsto[\varphi]_{\Delta(\mu)}$. We call $\Delta(\mu)$ the congruence theory implied by $\mu$. Given sequent theories $\Gamma \subseteq \Delta \subseteq \Delta(\mu)$, map $\nu\left([\varphi]_{\Delta}\right)=\mu\left([\varphi]_{\Gamma}\right)$ is the unique function (and Visser algebra morphism) that makes the following diagram commute.

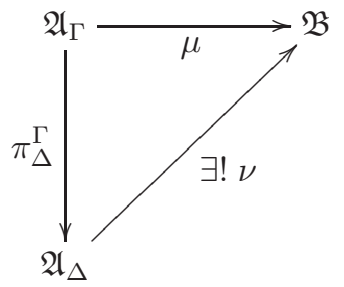


where $\nu$ is an isomorphism exactly when $\Delta=\Delta(\mu)$.

Given element $a$ of Visser algebra $\mathfrak{A}$, we have $R^{a}(\mathfrak{A}) \subseteq A$. So each function $\mu$ from $\mathfrak{A}$ uniquely determines a restricted function $\mu_{a}$ from $\mathfrak{R}^{a}(\mathfrak{A})$. Let $\mu: \mathfrak{A} \rightarrow \mathfrak{B}$ be a Visser algebra morphism. Then the following diagram commutes, with $\mu_{a}\left(x^{a a}\right)=\mu(x)^{\mu(a) \mu(a)}$.

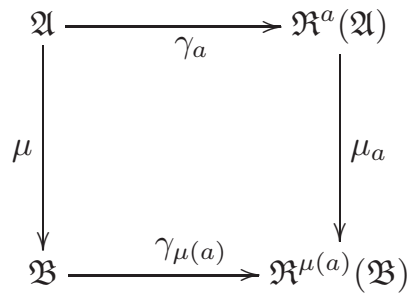

The following is not immediately self-evident since the idempotent onto maps $\gamma_{a}$ and $\gamma_{\mu(a)}$ need not be Visser algebra morphisms.

Proposition 4.5. Let a be element of Visser algebra $\mathfrak{A}$, and $\mu: \mathfrak{A} \rightarrow \mathfrak{B}$ be a Visser algebra morphism. Then $\mu_{a}$ is a Visser algebra morphism.

Proposition 4.6. Let $\Gamma$ be a sequent theory. Then the congruence theory implied by $\gamma_{0}: \mathfrak{A}_{\Gamma} \rightarrow$ $\mathfrak{R}^{0}\left(\mathfrak{A}_{\Gamma}\right)$ equals $\Gamma+\mathrm{CPC}$.

We write $\Gamma \vdash \varphi$ as short for $\Gamma \vdash(\top \Rightarrow \varphi)$. This agrees with default practice over IPC, where, with modus ponens, $\varphi \Rightarrow \psi$ and $\top \Rightarrow \varphi \rightarrow \psi$ are provably equivalent. So intuitionistic theories can ignore sets of sequents in favor of sets of formulas, by simply dropping the $\top \Rightarrow$ part.

Define $\xi(\varphi)$ as short for $((\top \rightarrow \varphi) \rightarrow \varphi) \rightarrow(\top \rightarrow \varphi)$. This is in agreement with the function $\xi$ over Visser algebras of the form $\mathfrak{A}_{\Gamma}$, since $\xi\left([\varphi]_{\Gamma}\right)=[\xi(\varphi)]_{\Gamma}$.

Theorem 4.7. Let $\Gamma$ be a sequent theory over BPC. Then for all formulas $\varphi$ we have

1. $\Gamma \vdash \varphi \rightarrow \xi(\perp)$ if and only if $\Gamma+\mathrm{CPC} \vdash \varphi \rightarrow \perp$

2. $\Gamma \vdash(\varphi \rightarrow \perp) \rightarrow \xi(\perp)$ if and only if $\Gamma+\mathrm{CPC} \vdash \varphi$

Over IPC, Theorem 4.7 reduces to the well-known:

Theorem 4.8 (Glivenko). Let $\Gamma$ be a theory over IPC. Then for all formulas $\varphi$ we have

1. $\Gamma \vdash \neg \varphi$ if and only if $\Gamma+\mathrm{CPC} \vdash \neg \varphi$

2. $\Gamma \vdash \neg \neg \varphi$ if and only if $\Gamma+\mathrm{CPC} \vdash \varphi$

\section{References}

[1] Majid Alizadeh And Mohammad Ardeshir. On the linear Lindenbaum algebra of basic propositional logic, Mathematical Logic Quarterly 50 no. 1 (2004), 65-70.

[2] Majid Alizadeh and Mohammad Ardeshir. On Löb algebras, II, Logic Journal of the IGPL 20 no. 1 (2012), 27-44.

[3] Mohammad Ardeshir. Aspects of Basic Logic. PhD Thesis, Department of Mathematics, Statistics and Computer Science, Marquette University, Milwaukee, 1995.

[4] Mohammad Ardeshir and Wim Ruitenburg. Basic propositional calculus I, Mathematical Logic Quarterly 44, no. 3 (1998), 317-343.

[5] Albert Visser. A propositional logic with explicit fixed points, Studia Logica 40 (1981), 155-175. 